\title{
Inventory Management subject to Multiplicative Inaccuracies
}

\author{
Selma KHADER ${ }^{1}$, Yacine REKIK ${ }^{2 *}$, Valérie BOTTA-GENOULAZ ${ }^{1}$, Jean- \\ Pierre CAMPAGNE ${ }^{1}$
}

\author{
${ }^{1}$ INSA Lyon, INSA-Lyon, DISP, Bâtiment Léonard de Vinci, 21 Avenue Jean Capelle, \\ 69621 Villeurbanne cedex, France \\ ${ }^{2}$ EMLYON Business School, DISP, 23 av. Guy de Collonges, 69134 Ecully, France
}

\section{Abstract}

The standard literature on inventory modeling is rarely differentiating between the inventory records and the physical inventory. In the recent years, some empirical studies highlighted that errors and inventory perturbations may occur in the inventory system. Such errors result in a gap between what the informational system shows and what is actually available for sales and used to satisfy the demand. The impact of such errors is particularly important in a wholesaling / e-retailing context where customer' demands are remotely satisfied based on the inventory records shown in the informational system. These errors could be modeled by an additive or multiplicative structure depending on the link between the error variability and the ordered quantity. The aim of the paper is to extend the existing literature by developing an inventory framework that permits the analysis and the performance improvement of an inventory system subject to a multiplicative errors setting. The multiplicative and stochastic settings known also as the stochastically proportional modeling of errors is not well developed in the literature despite the fact that such an assumption bears considerable association with reality. We provide comprehensive analytical and numerical studies and we also complete our contribution by a comparison between the additive and the multiplicative error settings where we derive interesting

\footnotetext{
* Corresponding author : email rekik@em-lyon.com, phone +33478337886
} 
managerial insights about the impact of wrongly modeling errors. We also focus on the benefit of applying our results compared with the case where errors are ignored or not known.

Keywords: inventory management, inventory inaccuracies, multiplicative errors, additive errors, e-retailing, wholesaling.

\section{Introduction}

The classical inventory management models assume implicitly that the physical stock available in a warehouse is equal to the quantity shown in the informational system, i.e.

- The quantity received from a supply system matches exactly the quantity ordered;

- The physical stock is not subject to perturbations (such as shrinkage, misplacement and damage) detected by the informational system;

- The quantity sold and delivered is accurately registered in the informational system;

- More generally, each change on the physical stock is well and accurately detected and updated in the informational system.

However, various factors may create a difference between the expected and the effective physical and informational flows and perturb their synchronized evolution. Raman, DeHoratius and Ton (2001), based on extensive empirical studies, revealed a discrepancy between recorded inventory and physical inventory. Such a difference, also known as the inventory inaccuracy, may be a major obstacle to the performance improvements for every firm (Kök and Shang 2007). The inventory inaccuracy sources can be classified as follows (Schardy, 1970; Atali, Lee, and Ozer, 2009):

- Inaccuracies resulting from a permanent shrinkage: some products could be deteriorated or stolen by customers in a store without being detected by the 
inventory manager and consequently without triggering necessary updates in the informational system.

- Inaccuracies resulting from temporary discrepancies: such errors could be a consequence of a misplacement which occurs when a fraction of the inventory is not stored in the right location in the store or in the warehouse.

- Inaccuracies resulting from transaction and record errors: such errors, occurring during inventory transactions (counting inventory, receiving an order or checking out at the register) impact mainly the Informational System (IS) inventory level while the Physical (PH) stock remains unchanged.

- Supply yield: in some particular cases, the production and/or the supply system could have a low yield. Subsequently, it could lead to inventory record inaccuracies because the error on the physical quantity could not be detected by the informational system.

In the context of warehouse / in-store retailer, the quantity shown in the IS (Informational System) does not play a major role in the process of demand satisfaction because the customer is physically present in the store, so his demand is confronted by the quantity available in the shelves. The situation is totally different in the context of wholesaling / e-retailing because the quantity available in the IS is used as an indicator to accept or to refuse the costumers' demands. Confronting the IS level and the received customers' demands generates a commitment which should normally be honored when shipping the products to the customers. Because of the inventory inaccuracy issue:

- The physical quantity could be lower than the quantity shown in the IS resulting in a situation where the commitment could not be honored and hence additional penalties could be charged to the wholesaler / e-retailer. 
- The physical quantity could be higher than the IS level. In such situation, some sales could be missed because the IS level has been underestimated and consequently some customers' demands have been rejected.

The inventory inaccuracy issue has been mainly studied in the recent years when exploring the impact of new and advanced identification technologies, such as the RFID (Radio Frequency Identification) technology, on supply chain tracking and tracing. The literature review on the inventory inaccuracy field is summarized in Table 1 under two main criteria: the inventory error source and the type of retailer (in-store retailer, eretailer)

\begin{tabular}{|c|c|c|c|c|c|}
\hline & $\begin{array}{l}\text { Permanent } \\
\text { shrinkage }\end{array}$ & $\begin{array}{l}\text { Temporary } \\
\text { discrepancies }\end{array}$ & $\begin{array}{l}\text { Transaction } \\
\text { and record } \\
\text { errors }\end{array}$ & Supply yield & $\begin{array}{l}\text { Set of } \\
\text { errors }\end{array}$ \\
\hline $\begin{array}{l}\text { in-store } \\
\text { retailer }\end{array}$ & $\begin{array}{l}\text { Xu et al.( } \\
2012) ; \\
\text { Rekik, } \\
\text { Sahin, and } \\
\text { Dallery } \\
\text { (2009); } \\
\text { Agrawal and } \\
\text { Sharda } \\
\text { (2012) } \\
\text { Fleisch and } \\
\text { Tellkamp } \\
\text { (2005); } \\
\text { DeHoratius, } \\
\text { Mersereau, } \\
\text { and Schrage } \\
\text { (2008) }\end{array}$ & $\begin{array}{l}\text { Ton and } \\
\text { Raman } \\
\text { (2010); } \\
\text { Rekik, Sahin, } \\
\text { and Dallery } \\
\text { (2009) }\end{array}$ & $\begin{array}{l}\text { Ighlehart } \\
\text { and Morey } \\
\text { (1972); } \\
\text { Sandoh and } \\
\text { Shimamoto } \\
\text { (2001); } \\
\text { DeHoratius } \\
\text { and Raman } \\
\text { (2008) }\end{array}$ & $\begin{array}{l}\text { Yano and } \\
\text { Lee (1995); } \\
\text { Inderfurth } \\
\text { (2004); } \\
\text { Kang and } \\
\text { Gershwin } \\
\text { (2005); } \\
\text { Rekik, } \\
\text { Sahin, and } \\
\text { Dallery } \\
\text { (2007); } \\
\text { Inderfurth } \\
\text { and } \\
\text { Vogelgesang } \\
\text { (2010) }\end{array}$ & $\begin{array}{l}\text { Heese } \\
\text { (2007); } \\
\text { Nachtmann, } \\
\text { Waller, and } \\
\text { Rieske } \\
\text { (2010); } \\
\text { Kök and } \\
\text { Shang, } \\
\text { (2007) }\end{array}$ \\
\hline $\begin{array}{l}\text { e- } \\
\text { retailer }\end{array}$ & & & & $\begin{array}{l}\text { Sahin and } \\
\text { Dallery } \\
\text { (2009) }\end{array}$ & $\begin{array}{l}\text { Rekik } \\
(2011)\end{array}$ \\
\hline
\end{tabular}

Table 1. Classification of inventory inaccuracy contributions

As shown it Table 1, with the exception of the investigations performed by Sahin and Dallery (2009) and Rekik (2011), the inventory inaccuracy problem has mainly been 
studied in the in-store retailing context. Sahin and Dallery (2009) and Rekik (2011) extended the inaccuracy issue by considering the wholesaling / e-retailing context where the demand satisfaction process is mainly based on the IS level.

The inventory inaccuracy literature may also be classified based on the assumed error structure and initial inventory equal to zero. If $Q$ denotes the order quantity and $Q_{I S}$ and $\mathrm{Q}_{\mathrm{PH}}$ denote the IS and the $\mathrm{PH}$ levels respectively, errors can be modelled based on:

- The additive error structure: $Q_{I S}$ and $Q_{P H}$ are written as $Q_{j}=Q+e_{j}, j=$ $\{I S, P H\}$ where $e_{j}$ is a random variable characterizing additive errors;

- The multiplicative error structure: $Q_{I S}$ and $Q_{P H}$ are written as $Q_{j}=\gamma_{j} Q, j=$ $\{I S, P H\}$ where $\gamma_{j}$ is a random variable characterizing multiplicative errors.

Transaction type errors (for example typing a 7 instead of 5 when entering an order or updating the informational system) could be modelled as additive because the error value is independent of the quantity $(Q)$ on the stock. In contrast, permanent shrinkage errors such as internal and external theft as well as damage could be modelled as multiplicative since the error magnitude might depend on the quantity on stock.

As mentioned earlier, Sahin and Dallery (2009) were among the first to contribute some research in the wholesaling / e-retailing context by means of developing a framework which is subject to uniformly distributed IS errors. Rekik (2011) extended this work by including both PH and IS errors under general error distributions on the assumption of an additive error form. Our work extends these two investigations in some very significant ways to include a multiplicative error structure impacting both on the IS and the PH inventory levels. In fact, our analytical results and in particular the associated managerial insights are totally different from (and sometimes contradictory to) those derived from the additive error structure developed by Rekik (2011). The aim of the paper is to 
complete analytically and managerially the inventory inaccuracy literature to cover the case of multiplicative errors under the e-retailing context. We also compare our results with existing ones and we particularly provide recommendation for practitioners about the modelling to adopt when errors structure is not clearly verified.

The remainder of our paper is organized as follows: in Section 2, we introduce and we formulate the framework under study. In Section 3, we present a fundamental result providing the optimal ordering policy and the conditions of its existence. In Section 4, we provide a numerical study that allows a sensitivity analysis associated with our multiplicative error structure model. In section 5, the optimal ordering strategies associated with the additive and the multiplicative error settings are illustrated and compared. Managerial insights, about the benefit of our results as well as the error modelling to adopt in case of a doubt about the error structure, are also provided. The paper ends with Section 6 which provides the conclusions and future research in the inventory inaccuracy field.

\section{The Framework under study}

Since the aim of the paper is to extend the investigation of Rekik (2011) to cover the multiplicative error setting under the e-retailing context, we consider the same framework assumptions: a single-product, single-period (newsvendor) problem in an e-retailing / wholesaling context subject to inventory inaccuracies with initial inventory quantity equal to zero. Figure 1 and the following sequence of events describe the way the inventory inaccuracy issue is modelled:

Insert Figure 1 here 
- Let $Q$ be the quantity that the inventory manager orders from the supply system. $Q$ is chosen in advance before the selling period based on an estimation of the random demand $D$, (1).

- The inventory manager receives from the supply system goods, updates the informational system by scanning products and stores them in the warehouse, (2).

- $\quad$ Because of errors, $Q_{P H}=\gamma_{P H} Q$, i.e. what is physically available in the warehouse, may not be equal to $Q_{I S}=\gamma_{I S} Q$, i.e. what the informational system shows as being available for sales, (3).

- During the selling single-period, the inventory manager receives the cumulative online orders from the final customers. $D$ denotes the demand received remotely from the customers. The inventory manager compares $D$ with the IS inventory record $Q_{I S}$ in order to accept or decline orders. If the total demand $D$ is lower than $Q_{I S}$, he accepts all the orders. Otherwise, he only accepts orders summing up to the IS inventory. Because of the e-retailing / wholesaling context, demand satisfaction is achieved based on the IS level and a commitment $K=$ $\operatorname{Min}\left(D, Q_{I S}\right)$ is performed during the selling period and before shipping the products to the customers, (4).

- At the end of the selling period, products will be delivered to the customers by a collective shipment. All the orders that the e-retailer has committed him/her self to should in principle be satisfied. However, this may not be always the case due to inventory inaccuracies. Such a situation occurs for instance when the demand $D$ is higher than $Q_{I S}$ and $Q_{I S}$ is higher than $Q_{P H}$. The quantity delivered to customer is written as Sales $=\operatorname{Min}\left(K, Q_{P H}\right)$, (5). 
The above sequence of events describes the multiplicative error structure. The additive one is exactly the same by writing $Q_{P H}$ and $Q_{I S}$ as $Q_{P H}=Q+e_{P H}$ and $Q_{I S}=Q+e_{I S}$ respectively.

In order to develop the optimal inventory policy of the multiplicative error structure and to compare it with the additive one, the notations used in this paper are as follows:

- D: the random variable representing the demand

- $f_{D}\left(\right.$ resp. $\left.F_{D}\right)$ : the PDF (resp. CDF ) characterizing D

- $\mu_{D}$ : the average of $D$

- $\sigma_{D}$ : the standard deviation of $D$

- $e_{I S}\left(\right.$ resp. $\left.e_{P H}\right):$ the random variable representing IS ( resp. PH)under the additive error setting

- $\gamma_{I S}\left(\right.$ resp. $\left.\gamma_{P H}\right)$ : the random variable representing IS (resp. PH)under the multiplicative error setting

- $\mu_{I S}^{A}\left(\right.$ resp. $\left.\mu_{P H}^{A}\right)$ : the average of $e_{I S}\left(\right.$ resp. $\left.e_{P H}\right)$

- $\sigma_{I S}^{A}\left(\right.$ resp. $\left.\sigma_{P H}^{A}\right):$ the standard deviation of $e_{I S}\left(\right.$ resp. $\left.e_{P H}\right)$

- $\mu_{I S}^{M}\left(\right.$ resp. $\left.\mu_{P H}^{M}\right):$ the average of $\gamma_{I S}\left(\right.$ resp. $\left.\gamma_{P H}\right)$

- $\sigma_{I S}^{M}\left(\right.$ resp. $\left.\sigma_{P H}^{M}\right)$ : the standard deviation of $\gamma_{I S}\left(\right.$ resp. $\left.\gamma_{P H}\right)$

- $D_{m}=D-e_{I S}:$ a random variable combining the demand and the IS additive error

- $f_{D_{m}}\left(\right.$ resp. $\left.F_{D_{m}}\right)$ : the PDF ( resp. CDF) characterizing $D_{m}$

- $e=e_{I S}-e_{P H}$ : the random variable that represents the difference between the IS and the $\mathrm{PH}$ additive errors

- $f_{e}\left(\right.$ resp. $\left.F_{e}\right)$ the PDF (resp. CDF) characterizing e

- $f_{I S}\left(\right.$ resp. $\left.F_{I S}\right)$ : the PDF (resp. CDF ) characterizing $\gamma_{I S}$

- $f_{P H}\left(\right.$ resp. $\left.F_{P H}\right)$ : the PDF (resp. CDF )characterizing $\gamma_{P H}$

- $\quad r$ : the unit selling price

- $\quad$ : the unit purchase cost

- $\quad$ : the unit salvage cost

- $\quad k$ : the unit cost paid for a nonsatisfied commitment 
- Q: the ordering quantity

- $Q_{A}^{*}$ : the optimal value of $Q$ under the additive setting

- $Q_{M}^{*}:$ the optimal value of $Q$ under the multiplicative setting

- $\pi^{A}$ : the expected profit function under the additive setting

- $\pi^{M}$ : the expected prof it function under the multiplicative setting

\section{The expected profit formulation}

Based on the sequence of events previously described the commitment $K$ and the Sales could be written as equations (1) and (2):

$$
\begin{gathered}
K=\operatorname{Min}\left(D, Q_{I S}\right) \\
\text { Sales }=\operatorname{Min}\left(K, Q_{P H}\right)
\end{gathered}
$$

For a given vector $\left(D, Q_{I S}, Q_{P H}\right)$, the profit achieved by the inventory manager could be deduced as follows (3):

$$
\text { Profit }=\text { r.Sales }+ \text { s. }\left(Q_{P H}-\text { Sales }\right)^{+}-c . Q_{P H}-k .\left(K-Q_{P H}\right)^{+}
$$

The first and the second terms of the profit function correspond to the margin achieved from sales and salvages respectively. The third term is associated with purchase costs and the last one corresponds to the penalty paid when a commitment is not satisfied, i.e., when $K>Q_{P H}$. By replacing $K$ and Sales by their expressions, the profit function (3) could also be written as equation (4):

$$
\begin{aligned}
& \text { Profit }=r \operatorname{Min}\left[\operatorname{Min}\left(Q_{I S}, D\right), Q_{P H}\right]+s\left[Q_{P H}-\operatorname{Min}\left(Q_{I S}, D\right)\right]^{+}-c Q_{P H}-k\left[\operatorname{Min}\left(Q_{I S}, D\right)-\right. \\
& \left.Q_{P H}\right]^{+} \\
& =(r-c) D-(r-c)\left[D-Q_{I S}\right]^{+}-(r-c+k)\left\{\left(Q_{I S}-Q_{P H}\right)-\operatorname{Min}\left[\left(Q_{I S}-D\right)^{+},\left(Q_{I S}-\right.\right.\right. \\
& \left.\left.\left.Q_{P H}\right)\right]\right\}+(c-s) \operatorname{Min}\left[\left(Q_{I S}-D\right)^{+},\left(Q_{I S}-Q_{P H}\right)\right]-(c-s)\left[Q_{I S}-D\right]^{+}
\end{aligned}
$$


Equation (4) can be interpreted as follows:

- $(r-c) D$ : represents the expected sales revenue;

- $-(c-s)\left[Q_{I S}-D\right]^{+}-(r-c)\left[D-Q_{I S}\right]^{+}+(c-s) \operatorname{Min}\left[\left(Q_{I S}-D\right)^{+},\left(Q_{I S}-Q_{P H}\right)\right]:$

represents the cost paid under an overstocking situation;

- $-(r-c)\left[D-Q_{I S}\right]^{+}$: represents the underage penalty incurred if a demand is not satisfied when answering customers' requests based on the IS level;

- $-(r-c+k)\left\{\left(Q_{I S}-Q_{P H}\right)-\operatorname{Min}\left[\left(Q_{I S}-D\right)^{+},\left(Q_{I S}-Q_{P H}\right)\right]\right\}$ : represents the additional (and more important) underage penalty incurred when a commitment is made but not honoured.

Based on the previous interpretations, we could define the following unit costs:

- $u_{1}=r-c$, the unit type 1 shortage cost paid when the IS level is not enough to satisfy a demand;

- $u_{2}=r-c+k$, the unit type 2 shortage cost paid when the PH level is not enough to satisfy a commitment;

- $\quad h=c-s$, the unit overage cost.

In the classical inventory literature where the inventory inaccuracy is not taken into account, only $h$ and $u_{1}$ are considered since the IS level is implicitly assumed to be equal to the PH inventory level.

Considering the multiplicative error structure $Q_{I S}=\gamma_{I S} Q$ (and $Q_{P H}=\gamma_{P H} Q$ ) and applying the expectation to the profit function permits us to deduce $\pi^{M}(Q)$ which is given by equation (5):

$$
\pi^{M}(Q)=(r-c) \mu_{D}-(c-s) \int_{\gamma_{I S}=0}^{+\infty} \int_{D=-\infty}^{Q \gamma_{I S}}\left(\gamma_{I S} Q-D\right) f_{D}(D) f_{I S}\left(\gamma_{I S}\right) d D d \gamma_{I S}
$$




$$
\begin{aligned}
& -(r-c) \int_{\gamma_{I S}=0}^{+\infty} \int_{D=-\infty}^{Q \gamma_{I S}}\left(\gamma_{I S} Q-D\right) f_{D}(D) f_{I S}\left(\gamma_{I S}\right) d D d \gamma_{I S} \\
& -(r-c+k) Q \int_{\gamma_{P H}=0}^{+\infty} \int_{\gamma_{I S}=0}^{+\infty}\left(\gamma_{I S}-\gamma_{P H}\right) f_{I S}\left(\gamma_{I S}\right) f_{P H}\left(\gamma_{P H}\right) d \gamma_{I S} d \gamma_{P H} \\
& +(r-s+k)\left(\begin{array}{c}
\int_{\gamma_{P H}=0}^{+\infty} \int_{\gamma_{I S}=0}^{\gamma_{P H}}\left(\gamma_{I S}-\gamma_{P H}\right) Q f_{I S}\left(\gamma_{I S}\right) f_{P H}\left(\gamma_{P H}\right) d \gamma_{I S} d \gamma_{P H} \\
+\int_{\gamma_{I S}=0}^{+\infty} \int_{\gamma_{P H}=0}^{\gamma_{I S}} \int_{D=Q \gamma_{I S}}\left(\gamma_{I S}-\frac{D}{Q}\right) Q f_{D}(D) f_{I S}\left(\gamma_{I S}\right) f_{P H}\left(\gamma_{P H}\right) d D d \gamma_{I S} d \gamma_{P H} \\
+\int_{\gamma_{I S}=0}^{+\infty} \int_{\gamma_{P H}=0}^{\gamma_{I S}} \int_{D=0}^{Q \gamma_{P H}}\left(\gamma_{I S}-\gamma_{P H}\right) Q f_{D}(D) f_{I S}\left(\gamma_{I S}\right) f_{P H}\left(\gamma_{P H}\right) d D d \gamma_{I S} d \gamma_{P H}
\end{array}\right)
\end{aligned}
$$

\section{The optimal inventory policy}

When employing a numerical analysis, the concavity of the expected profit function is not always verified. The aim of this section is to provide the conditions of the existence of an optimal ordering policy permitting to maximize the expected profit.

For this purpose let's first define the following two parameters $C 1$ and $C 2$ represented respectively in equation (6) and (7)which could be easily calculated using the unit costs as well as the errors’ parameters (average and standard deviation):

$$
C 1=\left(u_{2}+h\right) \int_{\gamma_{I S}=0}^{+\infty} \int_{\gamma_{P H}=0}^{\gamma_{I S}}\left(\gamma_{P H}-\gamma_{I S}\right) f_{P H}\left(\gamma_{P H}\right) f_{I S}\left(\gamma_{I S}\right) d \gamma_{P H} d \gamma_{I S}-\left(u_{1}+\right.
$$

h) $\int_{0}^{+\infty} \gamma_{I S} f_{I S}\left(\gamma_{I S}\right) d \gamma_{I S}$

$$
\begin{aligned}
& C 2=-u_{1}\left(\mu_{I S}^{M}\left(1-F_{I S}(0)\right)+f_{I S}(0) \sigma_{I S}^{M^{2}}\right)+u_{2}\left(\mu_{I S}^{M}-\mu_{P H}^{M}\right)-\left(u_{2}+h\right) \int_{\gamma_{P H}=0}^{+\infty}\left[\left(\left(\mu_{I S}^{M}-\right.\right.\right. \\
& \left.\left.\left.\gamma_{P H}\right)\left(F_{I S}\left(\gamma_{P H}\right)-F_{I S}(0)\right)-\sigma_{I S}^{M^{2}}\left(f_{I S}\left(\gamma_{P H}\right)-f_{I S}(0)\right)\right) f_{P H}\left(\gamma_{P H}\right) d \gamma_{P H}\right]
\end{aligned}
$$

\section{Theorem: the optimal ordering policy under the multiplicative structure}

The optimal ordering quantity $Q_{M}^{*}$ maximizing the expected profit function when errors are taken into account should satisfy the following equation (8):

$$
\int_{0}^{+\infty} \int_{0}^{\gamma_{I S}}\left[\left(\begin{array}{c}
\left(u_{2}+h\right) \gamma_{I S} F_{D}\left(\gamma_{I S} Q_{M}^{*}\right) \\
-\left(u_{2}+h\right) \gamma_{P H} F_{D}\left(\gamma_{P H} Q_{M}^{*}\right) \\
-\left(u_{1}+h\right) \frac{\gamma_{I S} F_{D}\left(\gamma_{I S} Q_{M}^{*}\right)}{F_{I S}\left(\gamma_{I S}\right)-F_{I S}(0)}
\end{array}\right) f_{P H}\left(\gamma_{P H}\right)\right] f_{I S}\left(\gamma_{I S}\right) d \gamma_{P H} d \gamma_{I S}=C 2
$$


The last equation could have 2, 1 or 0 solution depending on the values taken by $C 1$ and C2:

\begin{tabular}{|c|c|c|c|}
\hline \multicolumn{2}{|c|}{ Condition $Q_{M}^{*}$} & $\begin{array}{c}\text { Nb. of } \\
\text { solutions }\end{array}$ & Optimality \\
\hline$\left(C_{1}-C_{2}\right) C_{2} \geq 0$ & $C_{2} \leq 0$ & 1 & Optimal \\
\hline$\left(C_{1}-C_{2}\right) C_{2} \geq 0$ & $C_{2} \leq 0$ & 1 & Not optimal \\
\hline$\left(C_{1}-C_{2}\right) C_{2} \leq 0$ & $C_{2} \leq 0$ & 0 or 2 & If 2 solutions the lowest solution is optimal \\
\hline$\left(C_{1}-C_{2}\right) C_{2} \leq 0$ & $C_{2} \geq 0$ & 0 or 2 & If 2 solutions the highest solution is optimal \\
\hline Other combinations & \multicolumn{3}{|c}{ No solution } \\
\hline
\end{tabular}

The proof of this theorem is given in Appendix. A

\section{A numerical analysis of the impact of the multiplicative errors on the order quantity}

The objective of this section is twofold:

- A sensitivity analysis is performed in order to analyse of the impact of the different inputs parameters in particular the errors parameters $\left(\mu_{I S}^{M}, \mu_{P H}^{M}, \sigma_{I S}^{M}\right.$ and $\left.\sigma_{P H}^{M}\right)$ on the optimal ordering quantity.

- An analysis of the benefit of our contribution is shown thanks to the study of the gain obtained by using our optimal inventory policy compared to the case where errors are not taken into account (i.e. when the classical error free policy is used).

To do so, we consider two cost structures: products with a high (low, respectively) margin assuming that the shortage penalties are higher (lower, respectively) than the overage costs. Based on (Atali, Lee, and Ozer, 2009; Lee and Özer,2007; Kök and Shang 2007), we consider a normally distributed demand with average $\mu_{D}=20$ and a standard deviation $\sigma_{D}=4$. Table 2 provides the values of the different unit costs used to illustrate the high and low margin cases in our numerical study: 


\begin{tabular}{|l|l|}
\hline High Margin & Low Margin \\
\hline $\mathrm{c}=2$ & $\mathrm{c}=2$ \\
\hline $\mathrm{s}=1$ & $\mathrm{~s}=1$ \\
\hline $\mathrm{r}=20$ & $\mathrm{r}=2.5$ \\
\hline $\mathrm{K}=0 ; 5 ; 10$ & $\mathrm{~K}=0 ; 0.1 ; 0.3$ \\
\hline
\end{tabular}

Table 2. Simulation control parameter value

The behaviour of $Q_{M}^{*}$ in relation to the average of the IS and PH errors for a high margin product, illustrated in Figures 2 and 3 respectively, shows that the ordered quantity decreases with the average of both errors. The same behaviour holds for the case of a low margin product. Such a result is intuitively expected; if the errors tend to increase the IS and the PH levels, the inventory manager should decrease his ordered quantity.

\section{Insert Figures 2and 3 here}

By setting $\mu_{I S}^{M}=\mu_{P H}^{M}=1$ we illustrate in Figure 4 (Figure 5, respectively) the behavior of the optimal ordering quantity with $\sigma_{I S}^{M}$ under a high (low, respectively) margin product configuration.

When the variability of the IS error increases:

a) For products with a high margin, the optimal ordered quantity should increase to tackle the increasing inaccuracy variability in order to avoid shortage situations which are more penalizing than overage ones.

Insert Figures 4 here

b) For products with a low margin, the optimal ordered quantity increases in a first instance to avoid shortage situations resulting from errors. The curve slope changes then its direction and $Q_{M}^{*}$ becomes a decreasing function with the IS error variability. Such a slope change could be explained as follows: if errors are more 
important, increasing the ordered quantity contributes to the increase of the inaccuracy issue since errors are multiplicative $Q_{j}=Q+e_{j}, j=\{I S, P H\}$, as a consequence, even the physical stock increases and the probability of overstock situations is more important. Under a low margin product configuration, overstocking is more penalizing and consequently, decreasing the ordering quantity is a way to decrease the overstock probability.

Insert Figures 5 here

The same behaviour could also be observed and similarly explained when we study the behaviour of the optimal ordering quantity with the variability of the PH error.

Thanks to the last numerical study, it could already be shown that extending our signle period problem into the multi-period setting will not be analytically easy since the behaviour of the optimal ordering quantity is not monotonous with the error parameter. As a consequence a myopic policy will not be optimal under the multi-period setting.

We continue our numerical analysis with showing the added value of our results to the practitioners when compared with the situation where errors are ignored or not taken into account.

In fact, we could compare the optimal expected profit of our framework with the case where errors are ignored. In such a case, the inventory manager uses the error free, i.e. the newsvendor, optimal ordering quantity as a decision variable: 


$$
Y_{0}=F^{-1}\left[\frac{u_{1}}{u_{1}+h}\right]
$$

When ordering $Y_{0}$ instead of $Y_{M}^{*}$, the expected profit resulting from ignoring errors is given by $\pi^{M}\left(Q_{0}\right)$.

The gain resulting from taking into account inaccuracies when ordering is also given as:

$$
\text { Gain }=\pi^{M}\left(Y_{M}^{*}\right)-\pi^{M}\left(Q_{0}\right) .
$$

Insert Figures 6 here

Fig. 6 illustrates the evolution of the gain with the IS (PH) average and standard deviation in the case of high margin (The case with a low cost structure provides the same insights). It is important to notice that their behaviour is a direct consequence of the evolution of $Y_{M}^{*}$. In fact, it could be remarked that $Y_{M}^{*}$ converges to $Y_{0}$ when $\mu_{I S}^{M}$ and $\mu_{P H}^{M}$ converge to 1 and $\sigma_{I S}^{M}$ et $\sigma_{P H}^{M}$ converge to 0 .

Concerning the evolution of Gain with the IS and the PH errors average, it could be observed that when errors average increase the gap between $\pi^{M}\left(Y_{M}^{*}\right)$ and $\pi^{M}\left(Y_{0}\right)$ decreases to tends to zero.

With regards to the evolution of the Gain with the variability of the IS and the PH errors, the gap between $Y_{M}^{*}$ and $Y_{0}$ is increasing with $\sigma_{I S}^{M}$ which results in a higher difference between $\pi^{M}\left(Y_{M}^{*}\right)$ and $\pi^{M}\left(Y_{0}\right)$.

\section{A comparison between the additive and multiplicative error settings}

In order to clearly show the added value of our analytical result compared to the existing ones, the behaviour of the optimal ordering strategies under the multiplicative error settings is compared to the additive error settings studied by (Rekik, 2011). Under 
such an error structure, $Q_{I S}=e_{I S}+Q$ (and $Q_{P H}=e_{P H}+Q$ ), the expected profit is given by (9):

$$
\begin{aligned}
& \pi^{A}(Q)=(r-c) \mu_{D}-(c-s) \int_{D_{m}=-\infty}^{Q}\left(Q-D_{m}\right) f_{D_{m}}\left(D_{m}\right) d D_{m}-(r-c) \int_{D=Q}^{+\infty}\left(D_{m}-\right. \\
& Q) f_{D_{m}}\left(D_{m}\right) d D_{m}-(r-s+k) \int_{e=0}^{+\infty}\left[e\left[1-F_{D_{m}}(Q)\right]-(r-s+k) \int_{e=0}^{+\infty}\left[e\left[1-F_{D_{m}}(Q)\right]+\right.\right. \\
& \left.\left.\int_{D_{m}=Q-e}^{Q}\left(e-\left(Q-D_{m}\right)\right) f_{D_{m}}\left(D_{m}\right)\right) d D_{m}\right] f_{e}(e) d e+(c-s) \int_{e=0}^{+\infty}\left[\int_{D_{m}=Q-e}^{Q}(e-\right. \\
& \left.\left.\left.\left(Q-D_{m}\right)\right) f_{D_{m}}\left(D_{m}\right)\right) d D_{m}\right] f_{e}(e) d e
\end{aligned}
$$

The optimal order quantity $Q_{A}^{*}$ should satisfy the following equation (10):

$$
(r-c) F_{D_{m}}\left(Q_{A}^{*}\right)-(r-c)+(r-s+k) \int_{e=0}^{+\infty}\left[F_{D_{m}}\left(Q_{A}^{*}-e\right)-F_{D_{m}}\left(Q_{A}^{*}\right)\right] f_{e}(e) d e=0
$$

Regardless of the technical and the mathematical differences, we focus in this section on the managerial insights based on a numerical analysis. It is important to note that the error configuration (additive or multiplicative) is not easy to guess and validate when analysing empirical data. Our comparative study is motivated by the necessity to provide insights on the impact of modelling wrongly the inaccuracy issue, i.e., modelling multiplicative errors as additive and vice versa.

To ensure a fair comparison, we assume that the same unit costs apply for both error configurations. We focus in our study on the standard deviations of the IS errors, i.e. $\sigma_{I S}^{M}$ and $\sigma_{P H}^{M}$, since the standard deviation of the physical errors has a symmetric impact on the ordering strategies. That is, the insights provided on the IS error variability hold for the PH error as well.

We also set $\mu_{I S}^{A}=\mu_{P H}^{A}=0$ and $\mu_{I S}^{M}=\mu_{P H}^{M}=1$ to isolate the uncertainties associated with errors. We anlayze the ordering strategies as a function of $\sigma_{I S}^{A}$ and $\sigma_{I S}^{M}$. Figures 6 and 7 illustrate the behavior of the optimal ordering quantity as well as the optimal associated expected profit in relation to the $\sigma_{I S}^{M}$ and $\sigma_{I S}^{A}$ respectively and under a low margin 
configuration. Figures 8 and 9 illustrate the same issue under a high margin product configuration.

It could be remarked that:

- Under both error structures and both product margin configurations, the expected profit decreases with the IS error variabilty. Such a result is intuitively expected since an inventory system with more variable errors is subject to more shortage and holding penalties.

- Under the high margin product configuration and for both error structures, the optimal order quantities are increasing with the error standard deviations. To tackle the inaccuracy issue, it is better to order more since a shortgae (type 1 or 2 ) is more penalizing than an overstock situation.

- Under a low margin configuration, the additive and the multiplicative errors setting do not provide the same order quantity behaviour. Under the additive setting, in the presence of more variable errors, the inventory manager decreases the ordering quantity to decrease overstocking which is more penalizing than the shortage situation (low margin cost configuration). Then for higher values of $\sigma_{I S}^{A}$, he orders more to limit the impact of errors on shortage situations. For the multiplicative errors (Figure 2), there is a change of slope permitting to limit the impact of the error variability (as explained in section 4).

Insert Figures 7, 8, 9 and 10 here

Next we provide an equivalence analysis between the additive and the multiplicative error settings. For a given vector, $\left(\sigma_{I S}^{M}, \sigma_{P H}^{M}\right)$ we calculate the associated optimal order 
quantity $Q_{M}^{*}$. Then, we derive the possible solutions $\left(\sigma_{I S}^{A}, \sigma_{P H}^{A}\right)$ solving $Q_{A}^{*}=Q_{M}^{*}$ and we derive for each possible solution the impact of wrongly modeling errors by calculating the profit ratio $R=\pi^{A}\left(Q_{A}^{*}\right) / \pi^{M}\left(Q_{M}^{*}\right)$.

The analysis is illustrated in the following Table 3:

\begin{tabular}{|c|c|c|c|c|c|}
\hline$\sigma_{I S}^{M}$ & $\sigma_{P H}^{M}$ & $Q_{A}^{*}=Q_{M}^{*}$ & $\sigma_{I S}^{A}$ & $\sigma_{P H}^{A}$ & $R$ \\
\hline \multirow{8}{*}{0,03} & \multirow{8}{*}{0,12} & \multirow{8}{*}{18,31} & 0,004633 & 0,0685 & 1,60105 \\
\hline & & & 0,02445 & 0,06451 & 1,60104 \\
\hline & & & 0,03653 & 0,05813 & 1,60103 \\
\hline & & & 0,04661 & 0,05141 & 1,60101 \\
\hline & & & 0,05265 & 0,04503 & 1,60100 \\
\hline & & & 0,05937 & 0,03596 & 1,60099 \\
\hline & & & 0,06373 & 0,02824 & 1,60098 \\
\hline & & & 0,06743 & 0,01951 & 1,6009 \\
\hline \multirow{5}{*}{0,03} & \multirow{5}{*}{0,08} & \multirow{5}{*}{27,49} & 0,1333 & 1,38 & 1,0047 \\
\hline & & & 0,5638 & 1,187 & 1,0046 \\
\hline & & & 0,8606 & 0,8309 & 1,0045 \\
\hline & & & 1,039 & 0,4747 & 1,0043 \\
\hline & & & 1,098 & 0,1482 & 1,0042 \\
\hline \multirow{9}{*}{0,03} & \multirow{9}{*}{0,14} & \multirow{9}{*}{29,04} & 0,2669 & 2,939 & 1,0084 \\
\hline & & & 0,8458 & 2,686 & 1,0084 \\
\hline & & & 1,321 & 2,286 & 1,0081 \\
\hline & & & 1,707 & 1,781 & 1,0078 \\
\hline & & & 1,9 & 1,41 & 1,0076 \\
\hline & & & 2,033 & 0,9645 & 1,0075 \\
\hline & & & 2,122 & 0,4895 & 1,0074 \\
\hline & & & 2,167 & 0,1482 & 1,0073 \\
\hline & & & 2,627 & 0,7122 & 1,0182 \\
\hline \multirow{10}{*}{0,2} & \multirow{10}{*}{0,05} & \multirow{10}{*}{30,4} & 0,5489 & 4,007 & 1,0085 \\
\hline & & & 0,9645 & 3,844 & 1,0084 \\
\hline & & & 1,321 & 3,621 & 1,0083 \\
\hline & & & 1,677 & 3,31 & 1,0082 \\
\hline & & & 2,063 & 2,879 & 1,0078 \\
\hline & & & 2,36 & 2,375 & 1,0077 \\
\hline & & & 2,671 & 1,677 & 1,0073 \\
\hline & & & 2,805 & 1,157 & 1,0072 \\
\hline & & & 2,879 & 0,7419 & 1,0071 \\
\hline & & & 2,924 & 0,2817 & 1,0070 \\
\hline
\end{tabular}

Table 3. Correspondence values 
The following remarks could be deduced:

- To find a quantity equivalence between both error structures (by solving the equation $\left.Q_{A}^{*}=Q_{M}^{*}\right)$, many combinations $\left(\sigma_{I S}^{A}, \sigma_{P H}^{A}\right)$ exist. Such a result is intuitively expected: for a given value of the vector $\left(\sigma_{I S}^{A}, \sigma_{P H}^{A}\right)$, we can find another combination of such vector (by increasing $\sigma_{I S}^{A}$ and decreasing $\sigma_{P H}^{A}$, for instance) permitting to get the same order quantity.

- Within the same combination results, it could be remarked that when increasing $\sigma_{I S}^{A}$ and decreasing $\sigma_{P H}^{A}$, the ratio $R$ decreases. Consequently, one could deduce that physical errors are more penalizing than IS ones. We recall that the physical errors result in an increase of the overstock probabilities as well as the increase of the non-satisfaction commitment. If a commitment is not satisfied, the type 2 shortage penalty (which is more important than the type $1, u_{2}=u_{1}+k$ ) is charged to the inventory manager.

- $\quad$ The ratio $R$ is always higher than 1 : the multiplicative errors are more penalizing than the additive ones. Such a result could be explained by the fact that the error variability has more weight under the multiplicative setting since it is multiplied by the order quantity in such an error structure.

- As a consequence of the last point: if the modeling of errors is not straightforward (errors could be modeled as additive or multiplicative based on empirical data), it is better for the inventory manager to adopt the multiplicative error modeling.

\section{Conclusions}

In this paper we studied the impact inventory inaccuracies on the performance of an inventory management system. We considered the wholesaling / e-retailing context where 
the demand satisfaction is mainly based on the inventory position shown in the informational system which could be subject to errors and might not represent the actual stock physically available. We extended existing investigations by considering the multiplicative error structure which could be used to model many error sources such as shrinkage and damage where the additive modelling does not apply. We provided an analytical optimal ordering solution for the problem under concern and most importantly, we deduced different, and consequently complementary, managerial insights concerning the impact of errors on the optimal ordering policy (compared to existing investigations). Then we compared the inventory performance of the additive and the multiplicative error settings and we remarked that the resulted impacts are different and if the modeling of errors is not straightforward, it is better for the inventory manager to adopt the multiplicative error modeling because the impact of multiplicative errors settings is more important than additive errors settings on the expected profit function.

The results provided in this paper could be extended in many manners to consider the impact of new identification technologies such as RFID, on an inventory system subject to inaccuracies. In particular, one could provide conditions on the new technology costs making its deployment cost effective.

Further, in our paper we studied a single period/single stage problem which is the first step before extending to multi-period multi-stage formulations.

For the multi-period extension, we have shown in this paper that the analytical development could not be easy because of the non-monotonous behavior of the ordered quantity with the error parameters. A possible way to extend the multiplicative error framework to the multi-period setting would be the use of an optimization approach based on the service levels and not on the cost minimisation. 
Concerning the extension of the framework to the multi-stage and decentralized supply chain, it would be interesting to study the impact of the errors on all the supply chain actors. The classical coordination contracts (such buyback and revenue sharing contracts) should be reviewed to include the inventory inaccuracy issue. Intuitively, one could imagine that errors occurring in the e-retail side are beneficial for his supplier because the ordered quantity is higher to tackle the errors.

\section{References}

Agrawal, P.M., and R. Sharda. 2012. "Impact of frequency of alignment of physical and information system inventories on out of stocks: A simulation study”. International Journal of Production Economics 136(1): 45-55.

Atali, A., H. Lee, and O. Ozer. 2009. "If the inventory manager knew: Value of RFID under imperfect inventory information”. Tech. rep., Graduate School of Business, Stanford University. URL available at http://ssrn.com/abstract=1351606.

DeHoratius, N., A. Mersereau, and L. Schrage. 2008. "Retail inventory management when recordsare inaccurate”. Manufacturing \& Service Operations Management10(2): 257-277.

DeHoratius, N., and A. Raman. 2008. "Inventory record inaccuracy: an empirical analysis”. Management Science, 54(4): 627-641.

Fleisch, E., and C.Tellkamp.2005.” Inventory inaccuracy and supply chain performance: a simulation study of a retail supply chain”. International Journal of Production Economics 95(3): 373-385.

Heese, H. 2007. "Inventory record inaccuracy, double marginalization and RFID adoption”. Production and Operations Management16 (5): 542-553. 
Iglehart, D.,and R. Morey. 1972. "Inventory systems with imperfect asset information”. Management Science18(8): 388-394.

Inderfurth, K,.and S. Vogelgesang. 2010. "Parameter determination for production/inventory control in the case of stochastic demand and different types of yield randomness”. In Proceedings of the16 th International Symposium on Inventories Research. Budapest.

Kang, Y., and S.B. Gershwin.2005.” Information inaccuracy in inventory systems: stock loss and stockout”. IIE Transactions 37(9): 843-859.

Kök, A. G., and H.K. Shang. 2007. "Replenishment and inspection policies for systems with inventory record inaccuracy”. Manufacturing \& Service Operations Management, 9(2): 185-205.

Lee, H. L. and Özer, Ö. 2007. "Unlocking the Value of RFID”. Production and Operations Management, 16(1), 40-64.

Nachtmann, H., M. Waller, and D.W. Rieske. 2010. “The impact of point of sale data inaccuracy and inventory record data errors”. Journal of Business Logistics 31(1): 149-158.

Raman, A., N. DeHoratius, and Z. Ton. 2001. “Execution: The missing link in retail operations”. California Management Review43 (3): 136-152.

Rekik, Y. 2011.” Inventory inaccuracies in the wholesale supply chain”. International Journal of Production Economics133: 172-181.

Rekik, Y., E. Sahin, and Y. Dallery. 2009.” Inventory inaccuracy in retail stores due to theft: An analysis of the benefits of RFID”. International Journal of Production Economics 118(1):189198.

Rekik, Y., E. Sahin, and Y. Dallery. 2007. “A comprehensive analysis of the newsvendor model with unreliable supply”. OR Spectrum29 (2): 207-233.

Sandoh, H., and H. Shimamoto. 2001. “A theoretical study on optimal inventory taking frequency for retailing”. Journal of Retailing and Consumer Services8(1): 47-52. 
Sahin, E., and Y. Dallery. 2009. "Assessing the impact of inventory inaccuracies within a newsvendor framework”. European Journal of Operational Research197 (3): 108-118.

Schrady, D. A. 1970. “Operational definitions of inventory record accuracy”. Naval Research Logistics Quarterly17: 133-142.

Ton, Z., and A. Raman. 2010. "The Effect of Product Variety and Inventory Levels on Retail Sales: A Longitudinal Study”.Production and Operations Management 19(5): 546-560.

Xu, J., W. Jiang, G. Feng, and J. Tian. 2012. “Comparing improvement strategies for inventory inaccuracy in a two-echelon supply chain”. European Journal of Operational Research 221(1):213-221.

Yano, C. A., and H.L. Lee. 1995. "Lot sizing with random yields: A review”. Operations Research,43: 311-334.

\section{Acknowledgments}

Funding for this project was provided by a grant from the Région Rhône Alpes, France.

\section{Annex A: Theorem of multiplicative settings}

The profit function is defined as Equation (A.1):

$$
\begin{aligned}
& \pi^{M}(Q)=u_{1} \mu_{D}-h \int_{\gamma_{I S}=0}^{+\infty} \int_{D=-\infty}^{Q \gamma_{I S}}\left(\gamma_{I S} Q-D\right) f_{D}(D) f_{I S}\left(\gamma_{I S}\right) d D d \gamma_{I S} \\
& -u_{1} \int_{\gamma_{I S}=0}^{+\infty} \int_{D=Q \gamma_{I S}}^{+\infty}\left(D-\gamma_{I S} Q\right) f_{D}(D) f_{I S}\left(\gamma_{I S}\right) d D d \gamma_{I S} \\
& -u_{2} Q\left(\mu_{I S}^{M}-\mu_{P H}^{M}\right)+\left(u_{2}+\right. \\
& h)\left(\begin{array}{c}
\int_{\gamma_{P H}=0}^{+\infty} \int_{\gamma_{I S}=0}^{\gamma_{P H}}\left(\gamma_{I S}-\gamma_{P H}\right) Q f_{I S}\left(\gamma_{I S}\right) f_{P H}\left(\gamma_{P H}\right) d \gamma_{I S} d \gamma_{P H} \\
+\int_{\gamma_{I S}=0}^{+\infty} \int_{\gamma_{P H}=0}^{\gamma_{I S}} \int_{D=Q \gamma_{P H}}^{Q \gamma_{I S}}\left(\gamma_{I S}-\frac{D}{Q}\right) Q f_{D}(D) f_{I S}\left(\gamma_{I S}\right) f_{P H}\left(\gamma_{P H}\right) d D d \gamma_{I S} d \gamma_{P H} \\
+\int_{\gamma_{I S}=0}^{+\infty} \int_{\gamma_{P H}=0}^{\gamma_{I S}} \int_{D=0}^{Q \gamma_{P H}}\left(\gamma_{I S}-\gamma_{P H}\right) Q f_{D}(D) f_{I S}\left(\gamma_{I S}\right) f_{P H}\left(\gamma_{P H}\right) d D d \gamma_{I S} d \gamma_{P H}
\end{array}\right)
\end{aligned}
$$

After some simplification of profit equation we obtain the final expression Equation (A.2)

$$
\begin{aligned}
& \pi^{M}(Q)=u_{1}\left(\mu_{D}-\mu_{D}\left(1-F_{I S}(0)\right)\right)-Q \int_{\gamma_{I S}=0}^{+\infty} \gamma_{I S}\left(\left(u_{1}+h\right) F_{D}\left(\gamma_{I S} Q\right)-u_{1}\right) f_{I S}\left(\gamma_{I S}\right) d \gamma_{I S}+ \\
& \left(u_{1}+h\right) \int_{\gamma_{I S}=0}^{+\infty}\left(\mu_{D} F_{D}\left(\gamma_{I S} Q\right)-\sigma_{D}^{2} f_{D}\left(\gamma_{I S} Q\right)\right) f_{I S}\left(\gamma_{I S}\right) d \gamma_{I S}-u_{2} Q\left(\mu_{I S}^{M}-\mu_{P H}^{M}\right)+ \\
& \left(u_{2}+h\right) Q\left(\int _ { \gamma _ { P H } = 0 } ^ { + \infty } \left(\left(\mu_{I S}^{M}-\gamma_{P H}\right)\left(F_{I S}\left(\gamma_{P H}\right)-F_{I S}(0)\right)-\sigma_{I S}^{M^{2}}\left(f_{I S}\left(\gamma_{P H}\right)-\right.\right.\right.
\end{aligned}
$$




$$
\begin{aligned}
& \left.\left.f_{I S}(0)\right)\right) f_{P H}\left(\gamma_{P H}\right) d \gamma_{P H}+\int_{\gamma_{I S}=0}^{+\infty}\left(( ( \gamma _ { I S } - \frac { \mu _ { D } } { Q } ) F _ { D } ( Q \gamma _ { I S } ) + \frac { \sigma _ { D } ^ { 2 } } { Q } f _ { D } ( Q \gamma _ { I S } ) ) \left(F_{P H}\left(\gamma_{I S}\right)-\right.\right. \\
& \left.\left.F_{P H}(0)\right)\right) f_{I S}\left(\gamma_{I S}\right) d \gamma_{I S}-\int_{\gamma_{I S}=0}^{+\infty} \int_{\gamma_{P H}=0}^{\gamma_{I S}}\left(\left(\gamma_{P H}-\frac{\mu_{D}}{Q}\right) F_{D}\left(\gamma_{P H} Q\right)+\right. \\
& \left.\left.\left.\frac{\sigma_{D}^{2}}{Q} f\left(Q \gamma_{P H}\right)\right)\right) f_{P H}\left(\gamma_{P H}\right) f_{I S}\left(\gamma_{I S}\right) d \gamma_{P H} d \gamma_{I S}\right)
\end{aligned}
$$

So $\frac{\partial \pi^{M}}{\partial Q}$ and $\frac{\partial^{2} \pi^{M}}{\partial Q^{2}}$ could be expressed respectively as Equation (A.3) and Equation (A.4)

$$
\begin{aligned}
& \frac{\partial \pi^{M}}{\partial Q}=\int_{0}^{+\infty} \int_{0}^{\gamma_{I S}}\left[\left(\begin{array}{c}
\left(u_{2}+h\right) \gamma_{I S} F_{D}\left(\gamma_{I S} Q\right) \\
-\left(u_{2}+h\right) \gamma_{P H} F_{D}\left(\gamma_{P H} Q\right) \\
-\left(u_{1}+h\right) \frac{\gamma_{I I} F_{D}\left(\gamma_{I I} Q\right)}{F_{P H}\left(\gamma_{I S}\right)-F_{P H}(0)}
\end{array}\right) f_{P H}\left(\gamma_{P H}\right)\right] f_{I S}\left(\gamma_{I S}\right) d \gamma_{P H} d \gamma_{I S}-C 2 \\
& \left.\frac{\partial^{2} \pi^{M}}{\partial Q^{2}}=\int_{0}^{+\infty}\left(\left(u_{2}+h\right)\left(F_{P H}\left(\gamma_{I S}\right)-F_{P H}(0)\right)-\left(u_{1}+h\right)\right) \gamma_{I S}{ }^{2} f_{D}\left(\gamma_{I S} Q\right)\right) f_{I S}\left(\gamma_{I S}\right) d \gamma_{I S}- \\
& \left(u_{2}+h\right) \int_{0}^{+\infty}\left[\int_{0}^{\gamma_{I S}}\left(\gamma_{P H}^{2} f_{D}\left(\gamma_{P H} Q\right)\right) f_{P H}\left(\gamma_{P H}\right) d \gamma_{P H}\right] f_{I S}\left(\gamma_{I S}\right) d \gamma_{I S}
\end{aligned}
$$

We remind that $C 1$ and $C 2$ represented respectively in equation A.5 and A.6

$$
\begin{aligned}
& C 1=\left(u_{2}+h\right) \int_{\gamma_{I S}=0}^{+\infty} \int_{\gamma_{P H}=0}^{\gamma_{I S}}\left(\gamma_{P H}-\gamma_{I S}\right) f_{P H}\left(\gamma_{P H}\right) f_{I S}\left(\gamma_{I S}\right) d \gamma_{P H} d \gamma_{I S}-\left(u_{1}+h\right) \int_{0}^{+\infty} \gamma_{I S} f_{I S}\left(\gamma_{I S}\right) d \gamma_{I S} \\
& C 2=-u_{1}\left(\mu_{I S}^{M}\left(1-F_{I S}(0)\right)+\sigma_{I S}^{M^{2}} F_{I S}(0)\right)+u_{2}\left(\left(\mu_{I S}^{M}-\mu_{P H}^{M}\right)\right)-\left(u_{2}+h\right) \int_{\gamma_{P H}=0}^{+\infty}\left[\left(\left(\mu_{I S}^{M}-\right.\right.\right. \\
& \left.\left.\left.\gamma_{P H}\right)\left(F_{I S}\left(\gamma_{P H}\right)-F_{I S}(0)\right)-\sigma_{I S}^{M^{2}}\left(f_{I S}\left(\gamma_{P H}\right)-f_{I S}(0)\right)\right) f_{P H}\left(\gamma_{P H}\right) d \gamma_{P H}\right]
\end{aligned}
$$

Calculating the first derivative of the expected profit function and setting it equal to zero permits us to deduce that the optimal ordering quantity $Q^{*}$, as well as the conditions of its existence.

The optimal ordering quantity maximizing the expected profit function when errors are taken into account should satisfy

$$
\frac{\partial \pi^{M}}{\partial Q}\left(Q_{M}^{*}\right)=0
$$

Based on the expression of $\frac{\partial \pi^{M}}{\partial Q}, C 1$ and $C 2$ we have

$$
\begin{gathered}
\lim _{Q \rightarrow 0}: \frac{\partial \pi^{M}}{\partial Q}=-C 2 \\
\lim _{Q \rightarrow+\infty}: \frac{\partial \pi^{M}}{\partial Q}=C 1-C 2
\end{gathered}
$$


In order to find the behavior of $\frac{\partial \pi^{M}}{\partial Q}$ we study $\frac{\partial^{2} \pi^{M}}{\partial Q^{2}}$. It's worth to note that these two functions are continuous.

For studying $\frac{\partial^{2} \pi^{M}}{\partial Q^{2}}$ we distinguished two cases:

Fist case:

We define assumption $\mathbf{H 1}$

H1 $\mu_{I S}^{M}+3 \sigma_{I S}^{M} \leq F_{P H}^{-1}\left(\frac{u_{1}+h}{u_{2}+h}+F_{P H}(0)\right)$

We suppose that $f_{\mathrm{IS}}\left(\gamma_{\mathrm{IS}}\right)=0$ if $\left\{\begin{array}{c}\gamma_{I S} \leq \mu_{I S}^{M}-3 \sigma_{I S}^{M} \\ \mu_{I S}^{M}+3 \sigma_{I S}^{M} \leq \gamma_{\mathrm{IS}}\end{array}\right.$ and so $\frac{\partial^{2} \pi^{M}}{\partial Q^{2}}$ could be written as equation A.7

$$
\begin{aligned}
& \left.\frac{\partial^{2} \pi^{M}}{\partial Q^{2}}=\int_{\mu_{I S}^{M}-3 \sigma_{I S}^{M}}^{\mu_{I S}^{M}+3 \sigma_{I}^{M}}\left(\left(u_{2}+h\right)\left(F_{P H}\left(\gamma_{I S}\right)-F_{P H}(0)\right)-\left(u_{1}+h\right)\right) \gamma_{I S}{ }^{2} f_{D}\left(\gamma_{I S} Q\right)\right) f_{I S}\left(\gamma_{I S}\right) d \gamma_{I S}- \\
& \left(u_{2}+h\right) \int_{0}^{+\infty}\left[\int_{0}^{\gamma_{I S}}\left({\gamma_{P H}}^{2} f_{D}\left(\gamma_{P H} Q\right)\right) f_{P H}\left(\gamma_{P H}\right) d \gamma_{P H}\right] f_{I S}\left(\gamma_{I S}\right) d \gamma_{I S}
\end{aligned}
$$

Under the assumption $\mathbf{H 1}, \frac{\partial^{2} \pi^{M}}{\partial Q^{2}}$ is a negative function and $\pi^{M}(Q)$ is a concave function.

The following figures (A.1) and (A.2) illustrate the values $\sigma_{I S}^{M}$ as a function of the ratio $x=u 2 / u 1$ and $y=h / u 1$ for $\mu_{I S}^{M}=0.8$ and $\mu_{I S}^{M}=1$ with $\mu_{P H}^{M}=1$ and $\sigma_{P H}^{M}=0.05$.

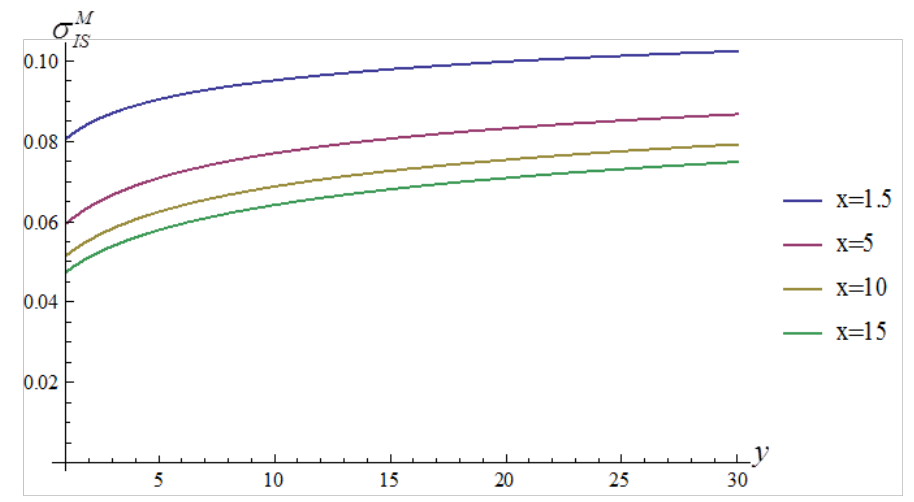

Figure A.1. $\sigma_{I S}^{M}$ function of $x$ et $y$ for $\mu_{I S}^{M}=0.8$

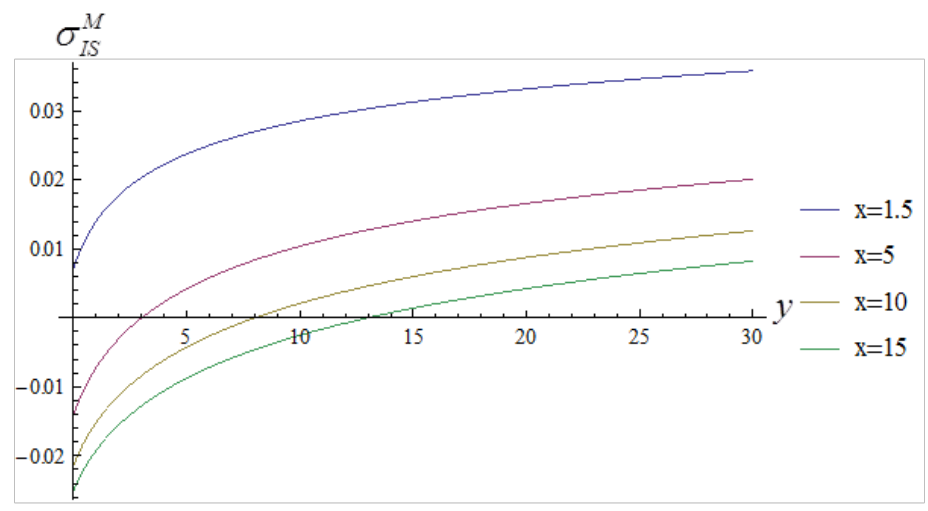

Figure A.2. $\sigma_{I S}^{M}$ function of $x$ et $y$ for $\mu_{I S}^{M}=1$

Second case: 
For any other combinations of inputs parameters we did numerical application with usual values. According to numerical applications $\frac{\partial^{2} \pi^{M}}{\partial Q^{2}}(Q)=0$ at the most one time. Table 1 represent the values used in numerical applications.

Table A.1. Values used in numerical applications

\begin{tabular}{|l|l|l|l|l|l|l|}
\hline$h$ & $u_{1}$ & $u_{2}$ & $\mu_{I S}^{M}$ & $\mu_{P H}^{M}$ & $\sigma_{I S}^{M}$ & $\sigma_{P H}^{M}$ \\
\hline$[1,10]$ & {$[0.1,20]$} & {$\left[u_{1}, 2 u_{1}\right]$} & {$[0.9,1.7]$} & {$[0.9,1.7]$} & {$[0.02,0.17]$} & {$[0.02,0.17]$} \\
\hline
\end{tabular}
depending on the values taken by $C 1$ and $C 2$.

Table A.2.Condition for $Q_{M}^{*}$

\begin{tabular}{|c|c|c|c|}
\hline \multicolumn{2}{|c|}{ Condition $Q_{M}^{*}$} & $\begin{array}{c}\text { Number } \\
\text { of } \\
\text { solutions }\end{array}$ & Optimality \\
\hline$\left(C_{1}-C_{2}\right) C_{2} \geq 0$ & $C_{2} \leq 0$ & 1 & Optimal \\
\hline$\left(C_{1}-C_{2}\right) C_{2} \geq 0$ & $C_{2} \geq 0$ & 1 & Not optimal \\
\hline$\left(C_{1}-C_{2}\right) C_{2} \leq 0$ & $C_{2} \leq 0$ & 0 or 2 & If 2 solutions the lowest solution is optimal \\
\hline$\left(C_{1}-C_{2}\right) C_{2} \leq 0$ & $C_{2} \geq 0$ & 0 or 2 & If 2 solutions the highest solution is optimal \\
\hline Other combinations & \multicolumn{3}{|c|}{ No solution } \\
\hline
\end{tabular}

